\title{
Screening and quantification of the tumor microenvironment with micro-ultrasound and photoacoustic imaging
}

\author{
The family of Vevo ${ }^{\circledast}$ Imaging Systems from FUJIFILM VisualSonics, Inc. is leading to translational \\ breakthroughs in the understanding of cancer progression and therapy. More specifically, high- \\ frequency ultrasound and photoacoustic imaging of orthotopic and patient-derived xenograft (PDX) \\ models of human cancer have empowered researchers in the past decade, with key translational \\ findings on tumor growth and microenvironment and in response to new and existing cancer \\ therapies. This Application Note focuses on the deep-tissue-imaging capabilities of micro-ultrasound \\ and photoacoustic imaging that contributed to these translational findings.
}

The Vevo Imaging Systems are the world's first commercially available high-frequency linear-array-based micro-ultrasound platforms. The family of products (Vevo 1100, 2100, 3100 and LAZR) has emerged as the gold standard in small animal in vivo imaging. The advanced system, Vevo LAZR, includes photoacoustics, a hybrid imaging modality that couples optical excitation with acoustic detection ${ }^{1}$.

The Vevo ultrasound systems operate at frequencies of 30-70 $\mathrm{MHz}$ (compared to frequencies of 3-15 MHz for clinical ultrasound systems), allowing 30- $\mu \mathrm{m}$ spatial resolution, required for detailed imaging of cancer models generated in mice, rats and zebrafish, among others. They are portable systems; do not emit ionizing radiation; and are configured and designed specifically for non-invasive in vivo imaging, quantification and longitudinalstudy data analysis. With these systems, cancer researchers are empowered with a composite of anatomical, functional, physiological and molecular data acquired simultaneously and sequentially in real time.

Each Vevo imaging system is connected to a series of applicationspecific transducers that transmit and receive non-invasive ultrasound signals to generate real-time images. The animal under study is anesthetized and maintained on a heated platform, and key physiological parameters such as heart rate, temperature, respiration and electrocardiogram readings are captured in real time and time stamped. An additional accessory is an injection mount that allows

\section{Minalini Lakshman and Andrew Needles}

FUJIFILM VisualSonics, Inc., Toronto, Ontario, Canada. Correspondence should be addressed to M.L. (mlakshman@visualsonics.com) or A.N. (aneedles@visualsonics.com) for ultrasound-guided precision in gene or drug delivery, as reported in immunotherapy studies of head and neck cancers.

Model system: orthotopic mouse model of human pancreatic cancer

Four-week-old female non-obese diabetic (NOD) severe combined immunodeficiency (SCID) mice $(n=2)$ were used for the surgical generation of orthotopic models of human pancreatic cancer, according to the University Health Network (UHN)-approved protocol for animal care and use. While the mice were under anesthesia, their abdominal skin and muscle were incised from the midline to allow for visualization and positioning of the pancreas lobes for direct implantation of human pancreatic cancer tissue. Successful implantation was confirmed using a dissection microscope, and mice were monitored as per the protocol approved by the Animal Care and Use Committee.

Method overview: non-invasive imaging of PDX pancreatic tumors on the Vevo LAZR

Image acquisition and quantitation were performed with the Vevo LAZR Imaging System (FUJIFILM VisualSonics, Inc.). This system combines micro-ultrasound and photoacoustic imaging into a common platform. Light generated from a tunable laser (680-970 $\mathrm{nm}$ ) was delivered through fiber optic bundles integrated into a lineararray ultrasonic transducer (LZ250, $f_{C}=21 \mathrm{MHz}$; LZ550, $\left.f_{C}=50 \mathrm{MHz}\right)$. Mice were imaged under isoflurane after implantation of pancreatic tumors (either $30 \mathrm{~d}$ or $15 \mathrm{~d}$ post-implantation). 


\section{What can micro-ultrasound and high-frequency photoacoustics do for oncology?}

In this Application Note, we focus on five different data sets on the tumor and its microenvironment obtained by using the associated imaging modes on the Vevo Imaging Systems. The Vevo LAZR provides all of the various micro-ultrasound and photoacoustic imaging modes required to fully assess the tumor microenvironment.

\section{Tumor sizing and quantitation in two and three dimensions with micro-ultrasound}

A 2D ultrasound of the abdominal cavity was acquired in brightness (B) mode in the transverse orientation (Fig. 1). The B-mode image is composed of regions of varying brightness or echogenicity. A prepalpable orthotopic pancreas tumor was identified $15 \mathrm{~d}$ postimplantation by its characteristic anatomy and size on ultrasound. This anatomy was defined by its depth below the skin line and its echogenicity relative to adjacent abdominal organs. The high frequency of $50 \mathrm{MHz}$ clearly defined the tissue margins for 3D reconstruction of the tumor and adjacent abdominal organs, in vivo and in minutes. Ultrasound of tumors with volumes of up to $300 \mathrm{~mm}^{3}$ has been reported in subcutaneous and orthotopic models in longitudinal studies addressing drug efficacies. Sastra and Olive ${ }^{2}$ have published a detailed method report on the imaging of orthotopic pancreas tumors in vivo.

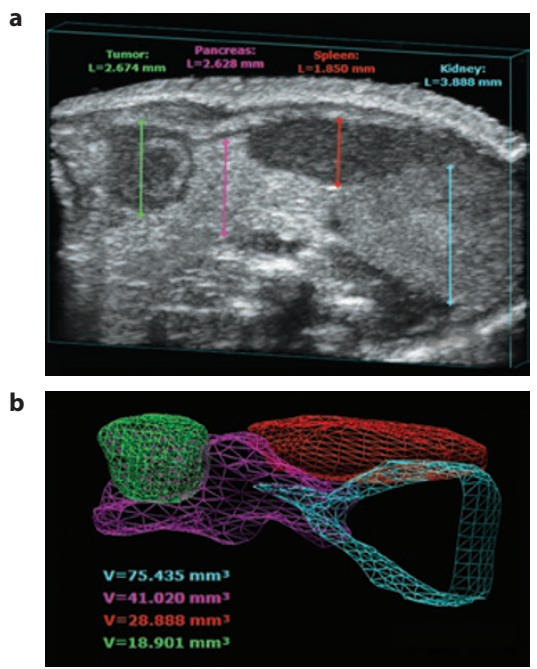

Figure 1 2D and 3D ultrasound images of prepalpable pancreas tumors $15 \mathrm{~d}$ after implantation into a mouse pancreas. L, length; $V$, volume.

\section{Microenvironment imaging of tumor angiogenesis and} tumor perfusion using contrast-enhanced ultrasound Additional characterization is accomplished by imaging and quantifying changes in the tumor microenvironment. Tumor vascular density and the functionality of microvessels as small as $3 \mu \mathrm{m}$ can be assessed using Doppler ultrasound imaging of slow blood flow (not shown) and contrast-enhanced ultrasound using gas-filled microbubbles (MicroMarker ${ }^{\circledR}$ ), respectively. In Figure 2a the filling of microbubbles into a pancreas tumor is depicted as a heat map that plots the contrast-enhanced ultrasound signal as a function of the wash-in rate of the microbubbles. Based on these data, it can be inferred that this tumor is well perfused at its periphery and poorly perfused at its core. Figure $\mathbf{2} \mathbf{b}$ is a non-invasive characterization of another pancreas tumor in which the microvascular distribution is clearly heterogeneous.
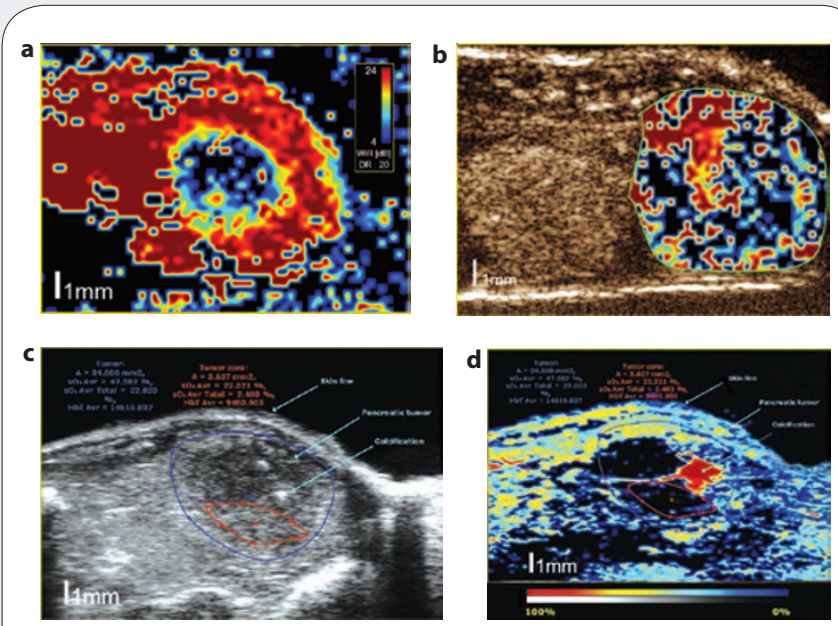

Figure $\mathbf{2}$ | Images of tumor microenvironment obtained with contrastenhanced ultrasound and photoacoustic imaging. (a,b) Heat maps of wash-in rate of gas-filled MicroMarker in two different pancreas tumors. Blue, low washin; red, high wash-in. (c,d) Heat maps of oxygen saturation in pancreas tumors from $\mathbf{a}$ and $\mathbf{b}$ in vivo. Blue, low oxygenation; red, high oxygenation (close to 100\%). A, area; $\mathrm{sO}_{2}$ Avr, average oxygen saturation; $\mathrm{HbT}$ Avr, average total hemoglobin concentration; WiR, wash-in rate.

\section{Microenvironment imaging of tumor hypoxia with} photoacoustic imaging

As reported recently ${ }^{3-5}$, tissue oxygenation-specifically, oxygen saturation consistent with hypoxia in the tumor-can be imaged and quantified in minutes. This characterization exploits the differing optical absorption of a dominant photoacoustic absorber called hemoglobin when it is oxygenated or deoxygenated. In fact, changing tissue vasculature is an aggressive phenotype in cancer progression ${ }^{6}$. Figure 2c shows an ultrasound image of the anatomy of an orthotopic pancreas tumor, and Figure 2d shows an oxygenation map of that tumor obtained using the Oxyhemo tool on the Vevo LAZR. This map characterizes the tumor as well oxygenated in its periphery and poorly oxygenated at its core. A rim of low oxygenation is evident at the interface of high oxygenation and no oxygenation. This rim was identified as positive for a hypoxic marker, as published in a separate report $^{7}$.

\section{Molecular imaging with micro-ultrasound and photoacoustics}

Contrast agents improve imaging by introducing a material into the bloodstream with acoustic or optical properties that differ from those of the tissues to be imaged. One commercially available molecular ultrasound agent is MicroMarker Target-Ready. This agent is coated with streptavidin and is ready for in vivo targeted imaging. Table 1 lists a selection of vascular markers for in vivo imaging with the targeted 
Table 1 | Molecular markers for imaging with ultrasound and photoacoustic contrast agents.

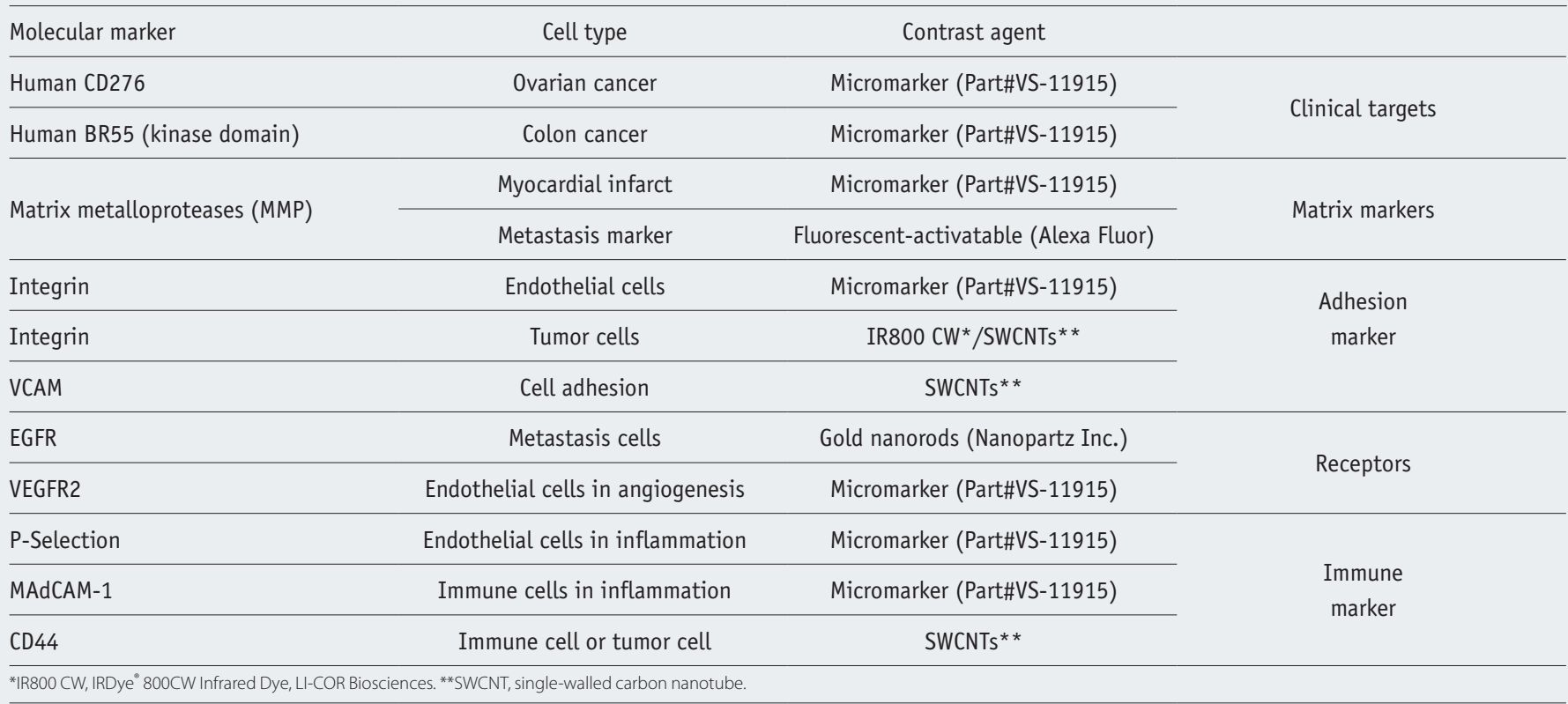

MicroMarker. The table also includes contrast agents that can be used for photoacoustic imaging of biomarkers such as markers of metastasis and stem cells, to name a few. Figure 3 shows spectrally unmixed molecular data obtained by photoacoustics that contrast with and simultaneously complement the positive nanoparticle signal detected by fluorescence.

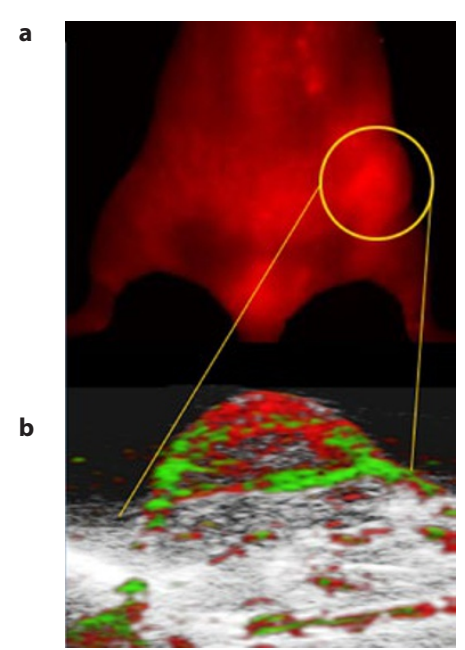

Figure $\mathbf{3}$ | What do the photoacoustic data of a taggable contrast agent look like? (a) A subcutaneous tumor positive for fluorescent signal from a nanoparticle. (b) The complementary photoacoustic data within the microenvironment of the tumor in $\mathbf{a}$. The taggable nanoparticle is spectrally unmixed as green and is present in the axial and depth peripheries of the tumor. Endogenous blood is clearly unmixed and is pseudocolored as red.

\section{Conclusions}

The Vevo Imaging Systems are the most comprehensive imaging solution in personalized and precision medicine. Many recent scientific insights on the relationship between functional tumor vasculature and onset of hypoxia were made possible by the combination of two or three of the described imaging modes, with high fidelity ${ }^{5,8}$. Such insights were obtained through deep-tissue imaging of orthotopic models of the colon and pancreas ${ }^{3,4,7}$. In addition,cancer genetics has recently benefited from photoacoustic imaging of melanin in reporter constructs in investigations of the role of oncogenes or tumor-suppressor genes ${ }^{9}$. The complexity of cancer research can be addressed by the fusion of all five data sets from one live scan of a cancer model, in real time and at high resolution.

\section{ACKNOWLEDGMENTS}

We are grateful to Drs. I. Lohse and D. Hedley for their kind gift of orthotopic models of pancreas cancer.

1. Needles, A. et al. Development and initial application of a fully integrated photoacoustic micro-ultrasound system. IEEE Trans. Ultrason. Ferroelectr. Frea. Control 60, 888-897 (2013).

2. Sastra, S.A. \& Olive, K.P. Quantification of murine pancreatic tumors by highresolution ultrasound. Methods Mol. Biol. 980, 249-266 (2013).

3. Gerling, M. et al. Real-time assessment of tissue hypoxia in vivo with combined photoacoustics and high-frequency ultrasound. Theranostics 4, 604-613 (2014).

4. Mallidi, S. et al. Prediction of tumor recurrence and therapy monitoring using ultrasound-guided photoacoustic imaging. Theranostics 5, 289-301 (2015).

5. Rich, L.J. \& Seshadri, M. Photoacoustic imaging of vascular hemodynamics: validation with blood oxygenation level-dependent MR imaging. Radiology http://dx.doi.org/10.1148/radiol.14140654 (2014).

6. Hanahan, D. \& Weinberg, R.A. Hallmarks of cancer: the next generation. Cell 144, 646-674 (2011)

7. Lakshman, M. et al. Molecular application of spectral photoacoustic imaging in pancreatic cancer pathology. In Proc. SPIE Meeting on Optics in Health Care and Biomedical Optics V (Ed. Luo, Q., Gu, Y. \& Li, X.D.) 855319 (SPIE, 2012).

8. Yin, M., Lakshman, M. \& Foster, F.S. Quantitative functional assessment of tumour microenvironment using contrast enhanced ultrasound and photoacoustic imaging. Proc. 2013 IEEE International Ultrasonics Symposium (Ed. IEEE Electrical Insulation Society Staff) 1833-1836 (IEEE, 2013).

9. Paproski, R.J. et al. Multi-wavelength photoacoustic imaging of inducible tyrosinase reporter gene expression in xenograft tumors. Sci. Rep. 4, 5329 (2014).

This article was submitted to Nature Methods by a commercial organization and has not been peer reviewed. Nature Methods takes no responsibility for the accuracy or otherwise of the information provided. 